Miroslava AUROVÁ

Universidad de Bohemia del Sur

\title{
LAS VARIANTES NOMINATIVAS DE LAS CONSTRUCCIONES NO NOMINATIVAS EN ESPAÑNL: DINÁMICA DEL SITEMA LINGÜÍSTICO
}

\section{Objetivo del artículo.}

El objetivo del presente artículo es ofrecer una imágen de la dinámica del sistema lingüístico que, gracias a sus características heredadas de los estadios anteriores del protoindoeuropeo, permite variantes opuestas dentro del comportamiento sintáctico nominativo. La aproximación tipológica se desarrolla a base del análisis de construcciones no-nominativas (impersonales).

\section{Estructura no nominativa.}

Español, como lengua indoeuropea, tiene una estructura de oraciones típicamente nominativa - o, en otras palabras, nominativo-acusativa. Esta es la estructura básica, con lo cual se entiende que la inmensa mayoría de las oraciones contiene, en su forma nuclear, un elemento nominal en caso nominativo, el sujeto, y un elemento nominal en caso acusativo, si está seleccionado por el verbo. El verbo, a su vez, adquire forma personal y concuerda con el sujeto en número y género (si la lengua tiene recursos para ello). La estructura nominativa puede ser representada con el esquema (1) y oraciones en (2): ${ }^{1}$

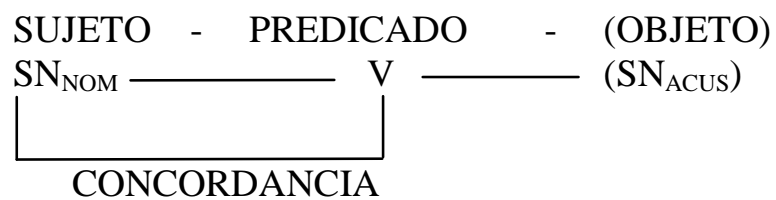

(2) a. [My husband $]_{\text {NOM }}$ has prepared [the dinner. $]_{\text {ACUS }}$ mi marido ha preparado la cena

b. $[\text { Todos }]_{\text {NOM }}$ hemos comido [la cena $]_{\text {ACUS }} \sin$ protestar

Sin embargo, existen lenguas con una estructura oracional básica diferente de la nominativa. Un ejemplo puede ser el vascuence, una lengua ergativa, que suele marcar al agente del evento y establecer una concordacia entre el predicado y sus argumentos de manera diferente de la de las lenguas nominativas. No obstante, las estructuras no nominativas (i.e. otras que nominativas) aparecen no sólo en lenguas primariamente ergativas o activas, sino también en lenguas nominativas, como son las indoeuropeas. Dado que tales estructuras no corresponden al

\footnotetext{
${ }^{1}$ Los términos y el concepto de definir las impersonales (construcciones no nominativas) sigue el modelo generativista, precisamente el iniciado y desarrollado por Noam Chomsky y conocido bajo el nombre de teoría de rección y ligamiento. Las impersonales se analizan en estudios (algunos ya clásicos dentro de este marco teórico) de Belletti (1982, 1987), BelletTi Y RizZi (1988), CinQue 1988, Miguel Aparicio Y Soriano (1988), Perlmutter (1978, 1983), Pollock (1981), Rizzi (1986). Dentro de la perspectiva minimista, véase por ejemplo FERNÁNDEZ SorIANO (1999), KempChinsky (2001), Masullo (1992, 1993), MendikoetXeA (1998, 2002), SuÑER (2002),
} 
esquema nominativo ofrecido en (1), podríamos llamarlas no nominativas. Otra razón para llamarlas así sería su rasgo sintáctico más importante, la ausencia de un elemento nominal marcado con nominativo. Hay que advertir que con la ausencia del sujeto no se entiende una mera no expresión del sujeto, como suele ser en casos de sujeto tácito, sino que se trata de una imposibilidad sistémica de insertar un elemento nominal nominativo en la posición del sujeto gramatical. Aun más, como consecuencia de la ausencia del sujeto, en tales estructuras falta concordancia entre el predicado y el elemento nominal nominativo. Veámos los siguientes ejemplos de diferentes lenguas europeas:

(3) a. Tui me, uxor, pudet. tu:GENme:ACUS esposa:VOC avergonzar:3SG «Tú me avergüenzas, mujer . (latín, PLAUT. As. 933)

b. Tak mu sie powiedziało. así le:DAT REFL decir:PRÉT.3SG.IMPRS «Así se le escapó de la boca. » (polaco, KURZOVÁ 1997, 1999)

c. Je mi stydno za bratra.

ser:3SG me:DAT avergonzar:NEU PREP hermano:ACUS

« Me avuergüenzo de mi hermano. »

d. B комнате пахнуло водкой.

en habitaciónLOC oler:3SG.NEU vodka:ABL

En la habitación olía a vodka.

e. Llueve. Prší (checo) It rains. Il pleut.

Estas oraciones tienen una característica común que se puede sumar en los siguintees tres puntos y representar en el esquema debajo:

(4) (i) ausencia de un argumento que si se realiza como sintagma nominal estará en nominativo

(ii) ausencia de la concordancia entre el sujeto y predicado

(iii) presencia de un argumento (facultativo) en caso oblicuo

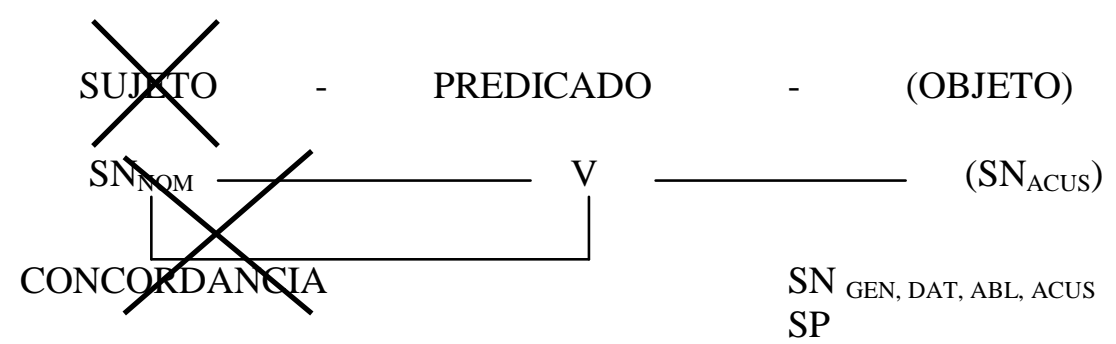

El específico comportamiento sintáctico de estas construcciones coincide con la idiosinrasia semántica, puesto que los verbos implicados en las construcciones no nominativas suelen denotar (i) fenómenos meteorológicos, (ii) experiencia psicológica, física y emocinal, y (iii), algún tipo de la modalidad. Un importante indicador semántico-sintáctico es la posibilidad de insertar un argumento que expresa el papel temático del experimentante del proceso o evento denotado por el verbo. Este argumento suele estar realizado como SN en caso dativo, pero también 
en genitivo o acusativo. Desde el punto de vista semántico el experimentante es una persona, un participante con rasgos [+HUMANO], que en el nivel sintáctico está desplazado de la posición de sujeto, típica de un agente, a la periferia sintáctica (casos oblicuos); como consecuencia, la posición de sujeto permanece no ocupada por una persona. Por esta razón las estructuras no nominativas percibieron un nombre tradicional de impersonales. Hay que advertir que los fenómenos sintácticos denominados oraciones impersonales y construciones no nominativas coinciden hasta cierto punto, sin embargo las denominaciones denotan construcciones diferentes y se usan en diferentes marcos teóricos. En nuestros trabajos concebimos impersonales y no nominativas como no sinónimas. ${ }^{2}$

\section{No nominativo versus nominativo.}

Las lenguas indoeuropeas, y por consiguiente el español, tienen la estructura básica nominativa, aunque permiten construcciones que no encajan en este sistema nominativo. Ahora se plantean dos preguntas: (1) ¿cuál es el origen de estas estructuras secundarias? (2) ¿cuál es su posición dentro del prevaleciente sistema nominativo, como en español?

En cuanto al origen de las construcciones no nominativas, deberíamos volver al estadio del protoindoeuropeo para el cual algunos autores suponen una estructuración diferente de la nominativa; una estructuración o ergativa o activa por una parte (conf. LEHMANN 1990, 1993, based specially on KLIMOV 1977 and GRAMKELIDZE \& IVANOV 1984, ${ }^{3}$ followed by DRINKA 1999 and DESZÖ 2001; also K.H. SCHMIDT 1979, BAUER 1995). Por otra parte, otras opiniones rechazan el carácter sistémico activo y ergativo del protouindoeuropeo (KURZOVÁ 1993, 1999; BAUER 2000) y, al contrario, suponen el patrón nominativo ya en protoindoeuropeo. Según estos, las lenguas indoeuropeas contienen rasgos de carácter ergativo o activo (p.ej. las $-s$ desinencias de los sustantivos animados de la tercera declinación en latín, véase KuRZOVÁ, 1993), pero carecen de estructuración sistémica ni ergativa ni activa. Sea como sea el patrón original del protoindoeuropeo, es obvio que un profundo análisis de construcciones no

\footnotetext{
${ }^{2}$ Cierta vaguedad del término impersonal se viene criticando desde hace mucho tiempo, ya que bajo el término suelen incorporarse construcciones sintácticamente diferentes. Tal heterogeneidad tiene un denominador común, la semántica, que hace reunir bajo la denominación impersonal oraciones en las que el participante humano está suprimido o eliminado, o simplemente pierde al papel de agente. Por consiguiente, en la heterogénea clase de impersonales encotramos oraciones sintácticamente sin sujeto (Llueve. No hubo muchos alborotos. Mihi pudet fratris.), pero también con sujeto (Uno no sabe. Mihi placet te videre. One should be polite.). Como consecuencia, han surgido diferentes propuestas de clasificar las oraciones impersonales en lenguas románicas, entre muchas citemos la de Llorente Maldonado (1972), de García Hernández (1992) y de Gómez Torrego (1994) dentro del marco funcionalista, la de NAPOLI (1973) dentro de la gramática generativa. Una interesante tipología de impersonales de lenguas europeas se ofrece en MORENO (1990). En nuestros trabajos (AUROVÁ (2005), tesis doctoral) preferimos usar el término no nominativo que implica su carácter sintáctico más evidente: la ausencia del sujeto. En este respecto el término no nominativo correspondía a oraciones sin sujeto (subjectless), sin embargo el término no nominativo parece ser más explicativo también en otro aspecto: las construcciones no nominativas paracen corresponder a una estructura no nominativa, probablemente heredada de los estadios anteriores del indoeuropeo.

${ }^{3}$ Las teorías sobre el tipo activo fueron objetivo de un análisis sistemático especialmente en la escuela de Moscú.
} 
nominativas contribuirá a la interpretación de estadios originales de lenguas indoeuropeas.

La cuestión de la posición de las estructuras no nominativas dentro de un sistema nominativo será respondida mejor a base del análisis de una lengua concreta, en nuestro caso de español. En los siguientes apartados veremos los tipos de construcciones no nomiantivas en español.

\section{Construcciones no nominativas de español}

Construcciones no nominativas típicas de la lengua española están definidas en el presente trabajo a base de rasgos sintácticos comunes: ausencia de un elemento nominal en la posición de subjeto, asignado con caso nominativo, regido por el verbo con el que, por consiguiente, concuerde. En la tabla que ofrecemos a continuación, se ven los tipos fundamentales de las construcciones que corresponden a esta característica sintáctica; las construcciones están ordenadas según las configuraciones sintácticas y según las realizaciones de los argumetnos. Debajo de la tabla aparece la característica del las construcciones. ${ }^{4}$

\section{Tabla 1}

\begin{tabular}{|c|c|c|c|c|c|}
\hline & VERBO & $\begin{array}{c}\text { TEMA } \\
{[\mathrm{V}, \mathrm{SN}]}\end{array}$ & $\begin{array}{c}\text { inserción del } \\
\text { Experimentante }\end{array}$ & TÍPICO DE & $\mathrm{n}^{\mathbf{0}}$ \\
\hline 1. & 3SG activo & --- & permitida & verbos meteorológicos & (5) \\
\hline 2. & 3SG activo & $\mathrm{SN}_{\text {acusativo }}$ & no permitida & $\begin{array}{l}\text { - haber existencial } \\
\text { - hacer meteorológico }\end{array}$ & $\begin{array}{l}\text { (6) } \\
(7)\end{array}$ \\
\hline 3. & 3SG activo & $\mathrm{SP}, \mathrm{Adv}$ & permitida & $\begin{array}{l}\text { - verbos de experincia } \\
\text { emocional o física } \\
\text { - verbos existenciales } \\
\text { - ser, hacerse y ponerse } \\
\text { (cambio de estado) }\end{array}$ & $\begin{array}{l}(8) \\
(9) \\
(10)\end{array}$ \\
\hline 4. & 3SG activo & $\mathrm{SP}+\mathrm{SC}$ & no permitida & hacer, intervalo temporal & $(11)$ \\
\hline 5. & 3SG activo & $\mathrm{SC}$ & $\begin{array}{l}\text { no permitida } \\
\text { permitida }\end{array}$ & $\begin{array}{l}\text { - verbos existenc., poner... } \\
\text { - verbos de experiencia } \\
\text { emocional y física } \\
\text { - verbo de elevación parecer }\end{array}$ & $\begin{array}{l}(12) \\
(13) \\
(14)\end{array}$ \\
\hline 6. & $\begin{array}{c}\text { SSG } \\
\text { activo+clítico } \\
S E\end{array}$ & $\mathrm{SN}_{\text {oblicuo }} / \mathrm{SC}$ & no permitida & - cualquier verbo & $(15)$ \\
\hline
\end{tabular}

3.1. El tipo de construcción de la primera línea en la tabla no contiente ningún SN que pueda ser asignado con caso nominativo. El verbo obtiene la forma de tercera persona de singular. Aunque falta un argumento con el papel temático de tema, puede ser insertado un experimentante. Típico de verbos meteorológicos.

(5) Nos anocheció en la carretera. (FERNÁNDEZ SORIANO \& TÁBOAS BAILÍN $2000: 1744)$

\footnotetext{
${ }^{4}$ Está claro que la lista de construcciones no pretende de ser exhaustiva, ni puede serlo por razones de espacio limitado. Por la misma razón hemos elegido de cada configuración sólo los verbos más importantes y más usados. Tampoco indicamos comportaminetos sintácticos excepcionales y construcciones raras. Una descripción más detallada ofrecemos en AUROVÁ (2005).
} 
3.2. El tipo de la segunda línea contiene un $\mathrm{SN}$ asignado con caso acusativo. El SN no concuerda con el verbo de 3SG. El marcado acusativo puede ser comprobado mediante un testo de pronominalización: si el SN está sustituído por un pronombre, éste adquire forma de clítico en caso acusativo. Típico del verbo existencial haber y del verbo meteorológico hacer.

(6) Había $3 \mathrm{SG}$ [ ss alumnos $]_{\mathrm{AC}}[\mathrm{sP}$ en el aula.]

(7) ...que $[\mathrm{sp} \text { a sus padres }]_{\mathrm{DAT}}[\mathrm{sN} \text { les }]_{\mathrm{DAT}}$ hizo $_{3: \mathrm{SG}}[\mathrm{sN} \text { buen tiempo }]_{\mathrm{AC}}$ durante su estancia en...

3.3. La construcción de la tercera línea contiene el predicado en la forma de tercera persona de singular y un argumento de presencia es obligatoria, realizado como SP o Adv. La concordancia entre el sujeto y el predicado falla. Típico de verbos de experiencia emocional o física, como oler, doler, picar, etc., verbos existenciales como faltar, sobrar, bastar, ocurrir, suceder, etc., y los verbos ser, hacerce y ponerse que denotan el cambio de estado.

(8) Me [vp pica 3 3SG [sp en el brazo]].

(9) Dice que basta $3 S_{S G}\left[{ }_{S P}\right.$ con [sN uno de los dos]].

(10) Se (nos) hace $3 \mathrm{SG}$ [sp de noche]

3.4. La cuarta línea presenta tipo de construcción compuesto de un verbo en tercera persona de singular con dos argumentos: complementos realizados como SSNN y SSCC. De nuevo, falta la concordancia entre el SN y el predicado. Típico del verbo hacer.

(11) Hace $_{3 S G}[\mathrm{NP}$ veinte años [CP que ocurrió.]]

3.5. La construcción de la línea quinta está compuesta del verbo en tercera persona de singular y un sintagma complementante o un sintagma tiempo. Típico de los verbos de comunicación (poner, constar, etc.), verbos de experiencia emocional y física (molestar, gustar, dar miedo, etc.) y verbos modales y de elevación, como parecer, convenir, importar, etc.

(12) Aquí pone $35 \mathrm{Sg}$ [sc que no se puede fumar.]

(13) Me molesta 3 SG $[$ cP que fumes.]

(14) Ocurre ${ }_{3 S G}$ [CP que el mundo está despertando.]

3.6. La construcción de la línea sexta contiene el verbo en tercera persona de singular, el clítico se de valor impersonal y, facultativamente, un SN que no concuerda con el predicado, pero es más bien el objeto de la acción denotada por el verbo. Es una posibilidad sistémica de formar impersonalidad (impersonales con se).

(15) Se cree $35 \mathrm{Sg}$ [sp en milagros.]

(16) Se avisó $_{3 S G}$ a $\left[{ }_{S N} \text { los bomberos. }\right]_{A C}$ 


\section{Varinates nominativas de las construcciones no nominativas}

Dado que el español es una lengua nominativa, su estructura sintáctica básica es la representada en (1). Pero acabamos de ver, que el español también permite estructuras diferentes de las nominativas, i.e. las no nominativas, que, pues, constituyen un estructura secundaria. Sin embargo, las construcciones que hemos visto en el apartado 3, excepcionales dentro del sistema -aunque productivas, pueden tener sus parejas cuyo comportamiento sintáctico sí cumple las características nominativas. Es decir, las construcciones no nominativas cambian a nominativas, si un SN asignado con caso nominativo está insertado, ocupando la posición de sujeto e interpretado, generalmente, como agente del proceso o evento denotado por el verbo. En las siguientes líneas veremos los ejemplos de variantes nominativas de las construcciones no nominativas:

4.1. En el caso verbos meteorológicos (3.1) un SN suele estar insertado en construcciones con significado figurado, metafórico. El SN concuerda con el predicado.

(18) Pareces un Greco. ¿Cómo pro $_{2 \mathrm{SG}}$ amaneciste $_{2 \mathrm{SG}}$ ? (BDS, Diego 164,14)

4.2. También las construcciones con el verbo haber y hacer (3.2.) tienen su pareja nominativa, aunque tales casos están restringidos a uso dialectal o muy coloquial:

(19) Hubieron ${ }_{3 \mathrm{PL}}[\mathrm{SN} \text { fiestas }]_{\mathrm{PL}}$ en el pueblo.

(20) Aquel año hicieron ${ }_{3 \mathrm{PL}}[\mathrm{sN} \text { unos calores terribles. }]_{\mathrm{PL}}$

4.3. Omitiendo la preposición de la construcción de tipo verbo+SP (3.3.), obtenemos una construccioń nominativa en la cual el verbo (imposible con ser, hacerse y poner de cambio de estado) concuerda con el SN:

(21) Basta 3 SG $[\text { sN uno de los dos. }]_{\mathrm{NOM}}$

(22) Me [vp pica [NP el brazo]].

4.4. También la construcción hace $+N P+C P$ (3.4) permite formar variante nominativa con el SN en la posición de sujeto, como se ve en (22). La misma situación ocurre con los verbos de comunicación del apartado 3.5., cuando el SN aparece en la posición de sujeto (23):

(23) Hacen $_{3 \mathrm{PL}}[\mathrm{sN} \text { dos años }]_{\text {NOM:PL }}$ que estaba.

(24) $\left[{ }_{\mathrm{sN}} \text { El texto }\right]_{\mathrm{NOM}}$ dice que ...

4.5. En el caso de los impersonales con se es posible formar la variante nominativa sólo con oraciones derivadas de verbos transitivos, en los que un SN ya está presente: de su posición de objeto pasa a la posición de sujeto: 
(25) Se avisaron 3 PL $[\text { sN } l o s \text { bomberos. }]_{\mathrm{NOM}: \mathrm{PL}}$

\section{Conclusiones}

Antes de llegar a una conclusión sobre la dinámica del sistema respecto a las construcciones no nominativas y sus variantes nominativas, sería oportuno reflexionar sobre la relación semántico sintáctica. Las construcciones no nominativas (y las impersonals en general) son un típico ejemplo de interferencia de la semántica en la sintaxis. Al examinar la característica semántica común a dichas construcciones, comprobamos que el agente, es decir el participante con rasgos [+HUMANO] está desplazado de la posición de sujeto sintáctico, designado al actor del proceso o evento, hacia la posición de un experimentante pasivo. En consecuencia, el experimentante es asignado con caso oblicuo. Tal comportamiento sintáctico probablemente comprueba que el protoindoeuropeo distinguía el principio de agentividad contra la aceptancia pasiva incorporado en la sintaxis. Es precisamente en este principio donde hay que buscar respuestas a las preguntas sobre las estructuración sintáctica original de los estadios más antiguos del protoindoeuropeo.

El principio de agentividad con un especial marcado del agente (lo cual podemos ver en las ya mencionadas terminaciones originales en $-s$ en latin en los nombres animados, por ejemplo) prevaleció en las lenguas indoeuropeas y fue extendido como una estructura general y no marcada, definida por Worf como Standard Averadge European. Sin embargo, al lado de esta estructura general, no marcada y, por lo tanto, primaria, nos encontramos con construcciones no nominativas. Es ahora cuando llegamos al dinamismo del sistema lingüístico. Aunque el sistema nominativo prevaleció y se mostró como primario, el patrón no nominativo, a su vez, se conservó como sistema paralelo y secundario; se conservó en unas clases de verbos específicas por una parte, y, por otra parte, como una herramienta sistémica (y considerablemente productiva) para expresar la no agentividad. Sin embargo, la fuerza del prevaleciente sistema nominativo llegó a la formación de construcciones nominativas con verbos originalmente involucrados en las construcciones no nominativas. Hemos visto que una lengua básicamente nominativa, aquí representada por el español, permite la formación de construcciones nominativas. Por otra parte, el mismo sistema de la misma lengua permite la formación de sus variantes nominativas. Este mecanismo puede ser representado con el siguiente esquema: 
(26) Dinamismo del sistema

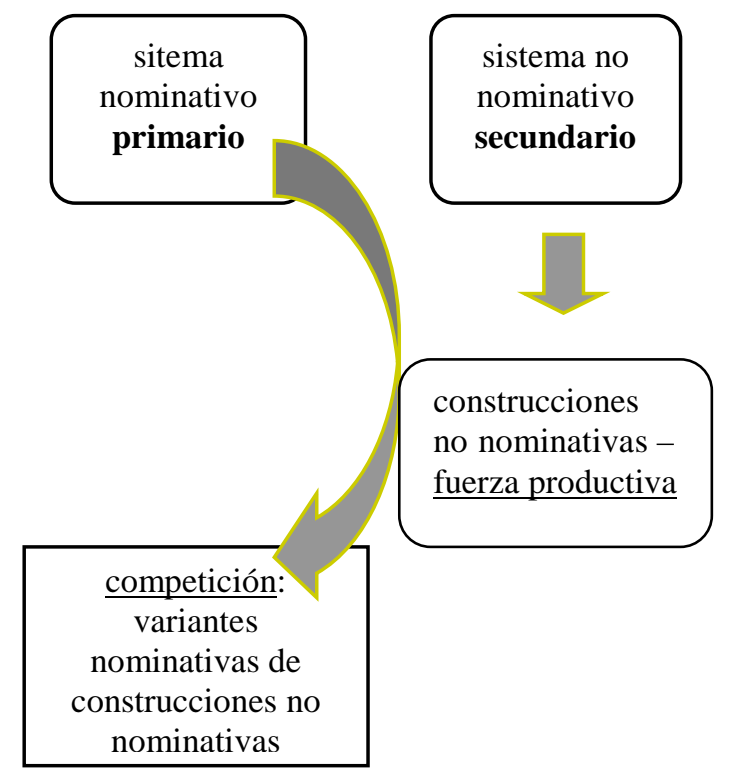

El esquema en (26) es válido para la perspectiva tanto diacrónica como sincrónica, ya que en el español actual encontramos construcciones no nominativas, interpretadas como estructuras secundarias dentro de un sistema primariamente nominativo. Tal dualismo tiene su origen en Latin, que también permitía variantes nominativas de construcciones no nominativas. Además, las construcciones no nominativas latinas corresponden, en general, a las españolas, por lo tanto podemos constatar que, aunque algunos tipos latinos desaparecieron y al contrario, nuevos se formaron, el sistema como tal ha permanecido en el sentido de que una lengua nominativa ofrece la posibilidad de tener construcciones no nominativas. Por otra parte, repetimos, ahora es cuando observamos la dinámica del sistema lingüístico: aunque se conservó, dentro de un sistema dominante, un sistema «secundario », que permite la foramción de estructuras no nominativas y que tiene su fuerza productiva, el potencial del sistema nominativo dominante está compitiendo con el no nominativo, formando varientes nominativas de las estructuras no nominativas.

Al final quisiera destacar una importancia tipológica de las construcciones no nominativas. Estas aparecen en diferentes lenguas europeas, pero cada lengua difiere en la extensión y abundancia de las construcciones no nominativas. Es precisamente la abundancia que podría servir de marcador tipológico, especialmente desde el punto de vista areal. Construcciones no nominativas abundan en las llamadas periferias del area lingüístico europeo, mientras que en el centro, caracterizado por el proceso de europeización con su preferencia por un sistema analítico, las construcciones no nominativas tienen una tendencia decresciente. 


\section{BIBLIOGRAFÍA}

AUROVÁ, Miroslava (2005), Non-nominative construcciones in Latin and Spanish. Tesis doctoral. Facultad de Filosofía, Universidad Carolina, Praga.

BAUER, Briggite L.M. (1995), The Emergence and Development of SVO Patterning in Latin and French. Diachronic and Psycholinguistic Perspectives. New York, Oxford. Oxford University Press. BAUER, Briggite (2000), Archaic Syntax in Indo-European. The Spread of Transitivity in Latin and French. Mouton de Gruyter.

BDS: Base de datos sintácticos. 〈www.bds.usc.es>

BelletTI, Adriana (1982), Morphological Passive and Pro-drop: the Impersonal Constructions in Italian, Journal of Linguistic Research 2:4, p. 1-33.

BelletTI, Adriana (1987), Los verbos psicológicos y la teoría temática, Sintaxis de las lenguas románicas. (Eds.) Demonte, Violeta \& M. Fernández Lagunilla, Madrid: El Arquero.

BelletTI, Adriana \& Luigi Rizzi (1988). «Psych-verbs and $\theta$-theory. » Natural Language and Linguistic Theory 6, p. 291-352.

CinQue, Guglielmo (1988), "On Si Constructions and the Theory of Arb.» Linguistic Inquiry 19:4, p. 521-581.

Deszö, László (2001), Two aspects of the study of animacy and case: A contribution to the long-range history of Russian and Serbian. Slovo a Slovesnost, LXII, 2, p. 105-114.

DRINKA, Bridget (1999), Alignment in Early Proto-Indo-European, Language Change and Typological Variation: In Honor of Winfred P.Lehman on the Occasion of His $83^{\text {rd }}$ Birtday. Vol.II: Grammatical Universals and Typology. (Eds.) Carol f. Justus \& Edgar C. Polomé. Journal of Indo-Eropean Studies Monograpf 31, p. 464-500.

FERNÁNDEZ SORIANO, Olga (1999a), Two types of impersonal sentences in Spanish: Locative and dative subjects, Syntax 2, p. 101-140.

FERNÁNDEZ SORIANO, Olga (1999b), Datives in Constructions with Unaccusative Se, CatWPL 7, 1999, p. 89-105.

FERnÁNDEZ SORIANO, Olga, TÁboAS BAILÍn, Susana (2000), Constructiones impersonales no reflejas, Gramática Descriptiva de la Lengua Española.I-III. (Eds.) Bosque Muñoz, Ignacio \& Violeta Demonte Barreto Bosque \& Demonte, Madrid: Espasa Calpe, p. 1723-1778.

GARCÍA HERnándEZ, B. (1990), Transitividad, intrnsitividad y causas de su desarrollo en latin trdío. Revista Española de Lingüística 20, p. 1-16.

GÓMEZ TORREGO, Leonardo (1994), La impersonalidad gramatical: descripción y norma. Madrid: Arco/Libros.

GRAMKELIDZE, Thomas, IVANOv, Vjacheslav V. (1984), Indoevropskij jazyk $i$ indoevropejcy: Rekonstrukcija $i$ istoriko-tipologičeskij analiz prajazyka $i$ protokul'tury. Vol.I. Tbilisi: Izdatel'stvo tbiliskogo univeriteta.

KEMPCHINSKY, P. (2001), Locative Inversion, PP topicalization and the EPP, Current Issues in Romance Languages, (Eds.) T. Sattefield et al. Netherlands: John Benjamins, p. 145-158.

KLIMOV, Georgij A. (1977), Tipologia jazykov aktivnogo stroja. Moscow: Nauka. 
KuRZOVÁ, Helena (1993), From Indoeuropean to Latin: the Evolution of a Morphosyntactic Type. Current Issues in Linguistic Theory, 104. Amsterodam/Philadelphia: John Benjamins Publishing Company.

KurzovÁ, Helena (1997), Morphosyntactic processes in Europe, Proceedings of LP'96. (Ed.) B. Palek, Prague: Charles University Press. p. 279-294.

KurzovÁ, Helena (1999), Syntax in the Indo-European Morphosyntactic Type, Language Change and Typological Variation: In Honor of Winfred P.Lehman on the Occasion of His $83^{\text {rd }}$ Birtday. Vol.II: Grammatical Universals and Typology. (Eds.) Carol f. Justus \& Edgar C. Polomé. Journal of Indo-Eropean Studies Monograpf 31. p. 501-520.

LEHMAN, Winfred P. (1990), Syntactic Residues, Language Typology 1987. Systematic Balance in Language. Amsterodam: Benjamins. p. 171-187.

LEHMANN, Winfred P. (1993), Theoretical Bases of Indo-European Linguistics. London: Routledge.

Llorente MALDONAdo DE GUEVARA, Antonio (1972), La expresión de la impersonalidad en español, Actas del Cuarto Congreso Internacional de Hispanistas, vol II. Salamanca, agosto de 1971. (Ed.) Eugenio de Bustos Tovar. Salamanca: Universidad de Salamanca.

Masullo, Pascual J. (1992), Quirky Datives in Spanish and the non-nominative subject parameter, MIT Working Papers in Linguisitcs 16: 82-1003

Masullo, Pascual J. (1993), Two types of quirky subjects: Spanish versus Icelandic, Proceedings of Nels 23, ed. by GLSA, p. 303-317. Amherst: University of Massachusetts.

MendiKoetXeA, A. (1998), Impersonal Constructions and the Theory of Grammar. Ms. U.A.M.

MendikoetXeA, Amaya (2002), La semántica de la impersonalidad, Las constructiones con se. (Ed.) Cristina Sánchez López Madrid: Visor Libros.

Miguel APARICIO, Elena de, FERnÁndeZ SoriAnO, Olga (1988), Proceso, acción y ergatividad: Las construcciones impersonales en castellano, Lenguajes naturales y lenguajes formales III, ed by $C$. Martín vide. Universidad de Barcelona.

MoRENO, Juan Carlos (1990), Processes and actions: internal agentless impersonals in some European Languages, Toward a Typology of European Languages. (Eds.) J.Bechert, G. Bernini \& C.Buridant. Mouton de Gruyter, Berlin/New York. p. 255-272.

NAPOLI, Donna Jo (1973), The two si's of Italian: An analysis of reflexive, inchoative and indefinite subject sentences in modern standard Italian. Unpublished doctoral dissertation, Harvard University Cambridge, Mass.

NAPOLI, Donna Jo (1976), At least two si's, Italian Linguistics. 2, p. 123-147.

PERLMUTTER, David M. (1978), Impersonals Passives and the Unaccusative Hypothesis, Proceedeing of the fourth Annual Meeting of th Berkeley Linguistics Society, (Ed.) J. Jaeger et al. (eds.) Berkeley, p. 157-189.

Perlmutter, David (1983), Personal vs. Impersonal Constructions, Natural Languageand LinguisticTheory 1(1983), p. 141-200.

POLLOCK, J.Y. (1981), On Case and impersonal constructions, Levels of syntactic representations, (Eds) R. May and Koster, Dordrecht: Doris. 
RIZZI, Luigi (1986), Null Subjects in Italian and the Theory of pro, Linguistic Inquiry 17, p. 501-557.

SCHMIDT, Karl Horst (1979), Reconstructing Active and Ergative Stages of PreIndo-European, Ergativity. Towards a Theory of Grammatical Relations. (Ed.) Frans Plank, London, New York: Academic Press, p. 333-345.

SUÑER, Margarita (2002), Las pasivas con «se » impersonal y la legitimación de las categorías vacías. Las construcciones con se, (Ed.) Sánchez López, Madrid, Visor Libros.

\begin{abstract}
Spanish is a language of nominative pattern, nevertheless some constructions show non-nominative syntactic behaviour. It is the case of constructions traditionally called impersonals, within GB framework, called non-nominative, i.e. constructions lacking overt nominative nominal element. Non-nominative character is usually explained as inheritance of ancient stage of I(ndo)-E(uropean) languages, suppressed by predominant subject-orientation of IE. In the current linguistic system of Spanish, some impersonal construction remain typically nonnominative, however, they can form nominative variants. On the basis of examples of Spanish impersonals, I will show the parametric variation in basic syntax behaviour, allowed by the systemic potentionality.
\end{abstract}

